\title{
Comment on An et al.: Plating osteosynthesis of mid-distal humeral shaft fractures: minimally invasive versus conventional open reduction technique
}

\author{
Anil Kumar Joshi • Saurabh Singh
}

Received: 3 March 2010 /Revised: 16 March 2010 /Accepted: 11 April 2010 /Published online: 11 May 2010

(C) Springer-Verlag 2010

\section{Dear Editor,}

We commend the excellent paper entitled "Plating osteosynthesis of mid-distal humeral shaft fractures: minimally invasive versus conventional open reduction technique" [1]. This innovation will definitely revolutionise our understanding of fracture care. In our experience minimally invasive plate osteosynthesis (MIPO) for mid-distal humeral shaft fractures, however, could be a surgically dangerous procedure because of the risk of radial nerve injury [2]. The humeral shaft fractures by the MIPO method approached anteriorly is a safer approach in our experience. Moreover, to reduce the risk of radial nerve injury, the forearm must be kept in full supination during plate insertion, and excessive force should be avoided during retraction of the lateral half of the brachialis muscle together with the radial nerve in the distal incision. The principle of biological fracture fixation and implementation of locking plates in traumatology is suitable for long bones such as femur and tibia. Moreover, in polytrauma patients the risk of fat embolism is minimised. The ultrasonographic study in MIPO done in humerus fractures postoperatively revealed that the implant is quite close to the radial nerve and make it vulnerable to traction injury [3]. The MIPO technique is not applicable to every fracture so absolute indications should be defined [4]. We would like to note that this technique requires experience, as there are variable danger zones for the musculocutaneous and radial nerves which should be determined as a percentage of the humeral length [5].

\section{References}

1. An Z, Zeng B, He X et al (2010) Plating osteosynthesis of middistal humeral shaft fractures: minimally invasive versus conventional open reduction technique. Int Orthop 34(1):131-135

2. Apivatthakakul T, Arpornchayanon O, Bavornratanavech S (2005) Minimally invasive plate osteosynthesis (MIPO) of the humeral shaft fracture: is it possible? A cadaveric study and preliminary report. Injury 36(4):530-538

3. Livani B, Belangero W et al (2009) Is MIPO in humeral shaft fractures really safe? Postoperative ultrasonographic evaluation. Int Orthop 33(6):1719-1723

4. Cole PA, Wijdicks CA (2007) The operative treatment of diaphyseal humeral shaft fractures. Hand Clin 23(4):437-448

5. Apivatthakakul T, Patiyasikan S, Luevitoonvechkit S (2010) Danger zone for locking screw placement in minimally invasive plate osteosynthesis (MIPO) of humeral shaft fractures: a cadaveric study. Injury 41(2):169-172

\footnotetext{
A. K. Joshi $(\bowtie)$

Department of Orthopaedics, VCSG Government Medical

Sciences \& Research Institute,

Srinagar Garhwal, Pauri Garhwal, Uttarakhand, India

e-mail: draniljoshi75@yahoo.co.in

S. Singh

Department of Orthopaedics, IMS BHU,

Varanasi, India
} 